\title{
A patient from Mexico with vaping-associated lung injury, seizures and renal failure
}

\author{
Alejandro E. Macias' ${ }^{1}$ Francisco J. Garcia², Sonia G. Saldana²
}

\section{Dear Editor,}

For vaping, a liquid is heated to create an inhalable aerosol. 'E-liquids' may contain nicotine, glycerol, nitrosamines, aldehydes, metals, organic compounds, phenolic compounds, polycyclic hydrocarbons, flavorings, alkaloids, and drugs; some of these may cause lung damage, particularly if adulterated ${ }^{1-3}$. In the US, the FDA has issued also an alert on vaping-associated seizures; the agency has received 127 reports of seizures that occurred between 2010 and $2019^{4}$. An outbreak of lung injuries associated with vaping has been reported with 2172 acute cases and 42 deaths, in the US. In about $86 \%$ of the cases, people reported use of products containing tetrahydrocannabinol (THC), many from street vendors ${ }^{3,5}$. Analyses of bronchoalveolar lavage fluid samples of patients with vaping-associated lung injuries has identified vitamin $\mathrm{E}$ acetate, an additive in some THC-containing products ${ }^{3}$.

We present here a case in Mexico of a previously healthy 31-year-old male with seizures and lung damage that meets the CDC definition for probable vapingassociated lung injury ${ }^{5}$. The patient used daily e-cigarettes, containing nicotine and THC, which he acquired online (in Mexico, vaping products are illegal). On 8 September 2018, his father found him unconscious; while being transferred to the hospital he suffered two generalized tonic-clonic seizures. At the emergency department, he was stuporous and required intubation and mechanical ventilation due to acute respiratory distress syndrome (Kirby index, $90 \mathrm{mmHg}$ ). His blood tests reported leukocytosis $(21000 / \mathrm{uL}$ with predominance of lymphocytes 11750/ uL, eosinophiles 430/uL, neutrophiles 6620/uL), aminotransferases mildly elevated (AST 45.7 U/L, ALT 102.4 U/L), serum glucose was $272 \mathrm{mg} / \mathrm{dL}$, and creatinine $1.3 \mathrm{mg} / \mathrm{dL}$. Arterial blood gas showed mixed high anion gap acidosis. The spun urine specimen showed a countless amount of erythrocytes. The lumbar tap found normal fluid and the head CT was normal. The chest x-ray showed opacity at the right lung base; the CT showed a right pleural effusion and a posterior-basal pulmonary infiltrate (Figure 1). He received anticonvulsive drugs, two doses of systemic steroids for the first 48 hours (equivalent to 50 and $25 \mathrm{mg}$ of prednisone), and antibiotics, although the clinicians did not believe that infection was the sole cause of the lung injury. After 72 hours, his serum creatinine reached $7 \mathrm{mg} / \mathrm{dL}$. During the following days he did not suffer from additional seizures, his respiratory condition improved, and his renal failure resolved with hydration only. He was extubated on the fifth hospitalization day. He is currently healthy.

The patient had suffered from seizures, renal failure, and lung injury temporarily associated with vaping. There may have been many similar but not reported cases outside US. It is appropriate that the health alert effective in the US be observed globally and that particular care is taken to avoid the use of substances acquired in informal commerce.

\author{
AFFILIATION \\ 1 Department of Medicine, \\ University of Guanajuato, \\ Leon, Mexico \\ 2 Department of Intensive \\ Care, Medica Campestre \\ Hospital, Leon, Mexico \\ CORRESPONDENCE TO \\ Alejandro E. Macias. \\ Department of Medicine, \\ University of Guanajuato, \\ 20 de Enero 929, Leon, \\ Guanajuato, 37000 Mexico. \\ E-mail: aaeemmhh@yahoo. \\ com \\ KEYWORDS \\ e-cigarette, vaping, disease \\ Received: 17 November 2019 \\ Accepted: 18 November 2019
}


Figure 1. A chest tomography showing a right-side effusion together with a posterior-basal infiltrate in a patient using E-cigarettes

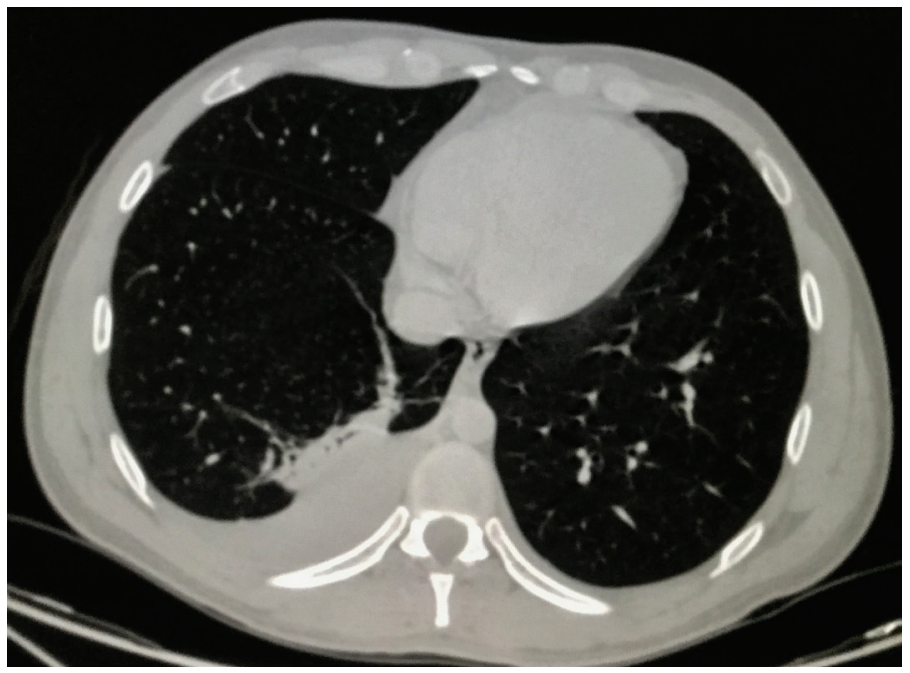

\section{REFERENCES}

1. National Academies of Sciences, Engineering, and Medicine. Public health consequences of e-cigarettes. Washington, DC: National Academies Press, 2018. doi:10.17226/24952

2. Layden JE, Ghinai I, Pray I, et al. Pulmonary illness related to e-cigarette use in Illinois and Wisconsin — preliminary report. N Engl J Med. 2019. doi:10.1056/NEJMoa1911614

3. Blount BC, Karwowski MP, Morel-Espinosa M, et al. Evaluation of bronchoalveolar lavage fluid from patients in an outbreak of e-cigarette, or vaping, product use-associated lung injury - 10 States, August-October 2019. MMWR Morb Mortal Wkly Rep. 2019;68:1040-1041. doi:10.15585/mmwr.mm6845e2

4. U.S. Food Drug Administration. FDA In Brief: FDA encourages continued submission of reports related to seizures following e-cigarette use as part of agency's ongoing scientific investigation of potential safety issue. https://www.fda.gov/ news-events/fda-brief/fda-brief-fda-encourages-continued-submission-reports-related-seizures-following-e-cigarette-use. Published August 7, 2019. Accessed November 11, 2019.

5. Centers for Disease Control and Prevention. Outbreak of Lung Injury Associated with the Use of E-Cigarette, or Vaping, Products. https://www.cdc.gov/tobacco/basic_information/e-cigarettes/severe-lung-disease.html. Updated November 14, 2019. Accessed November 14, 2019.

\section{CONFLICTS OF INTEREST}

The authors have completed and submitted the ICMJE Form for Disclosure of Potential Conflicts of Interest and none was reported.

\section{FUNDING}

There was no source of funding for this research. 